\title{
SIVB 2021: In Vitro OnLine, June 5 - 9, Virtual Meeting
}

\author{
Late Submission Abstracts
}

\section{ANIMAL SYMPOSIA ABSTRACTS}

ORGANOIDS: UNDERSTANDING AND DEVELOPING HUMAN DISEASE MODELS - Monday, June 7, 2021, 3:30 pm - 5:00 pm

A-11 Microphysiological Models of the Gut to Investigate Epithelial Physiology

S. Magness, University of North Carolina at Chapel Hill \& North Carolina State University

MODULATING THE GUT MICROBIOTA: APPLICATION OF PREBIOTICS AND PROBIOTICS FOR HUMAN HEALTH - Tuesday, June 8, 2021, 11:15 pm - 1:15 pm

A-14 The Pleiotropic Effects of Prebiotic Galacto-Oligosaccharides on the Aging Gut

M. Andrea Azcarate-Peril, University of North Carolina

\section{ANIMAL CONTRIBUTED PAPER SESSION ABSTRACTS}

IN VITRO ANIMAL CELL SCIENCES CONTRIBUTED PAPER SESSION - Monday, June 7, 5:15 pm - 6:30 pm

A-1007 Antiviral Innate Immune Responses Induced by DsRNA-Nanoparticle Complexes Inhibit Human Coronavirus Infections In Vitro

Stephanie J. Dewitte-Orr, Wilfrid Laurier University, Tamiru N. Alkie, and Shawna L. Semple

\section{ANIMAL POSTER ABSTRACTS}

CELL BIOLOGY, CELL \& TISSUE MODELS, AND INFECTIOUS DISEASES INTERACTIVE POSTER SESSION Tuesday, June 8, 2:15 pm - 3:15 pm

A-3000 Inhibition of TMA Production by (epi)catechin and Their Gut-derived Metabolites

Lisard Iglesias-Carres, North Carolina State University, Lyric K. Ramsue, Gabriella P. Agnello, Kathryn C. Racine, and Andrew P. Neilson

A-3001 Impacts of Plyinosinic/Polycytidylic Acid and Novel Phytoglycogen-based Nanoparticles on Immune Defense Agents and Receptors in Human Models

Kristof Jenik, Wilfrid Laurier University, Tamiru Alkie, Megan Dutcher, Emily Moore, Jondavid Dejong, and Stephanie J. DeWitte-Orr

A-3002 2D Colonoid Transwell Model of Human Colonic Adenoma

Kateryna Karpoff, Caroline L. Mccarthy, The University of Michigan Medical School, Ashley J. Cuttitta, Margaret S. Bohm, Michael K. Dame, and Jason R. Spence

A-3003 An In Vitro System for Maturation and Ovulation of African Catfish Ovarian Follicles

Nevena Kitanović, Hungarian University of Agriculture and Life Sciences, Zoran Marinović, Quyen Ngoc Nguyen, Tamás Müller, Balázs Kovács, Béla Urbányi, Gergely Bernáth, and Ákos Horváth

A-3005 Evaluation of the Effects of Cadmium on NRK-52E, HK-2, and RPTEC Proximal Tubule Renal Epithelial Cells Miloni Patel, Midwestern University, Kelly Keeler, Taulant Besiri, Rachel Arceneaux, Heshaam Latifi, Vishal Patel, Joshua R. Edwards, Walter C. Prozialeck, and Michael J. Fay

A-3006 Exploring DsRNAi in Human Cells as a Potential Antiviral Therapy for Coronaviruses Shawna L. Semple, Wilfrid Laurier University, and Stephanie J. DeWitte-Orr 


\section{CELL AND TISSUE MODELS}

A-3007 The Effect of Epithelial-Stromal Cross-talk on Tumor Cell Metabolism in Basal Cell Carcinoma Development

Tatiana Mendez, Tufts University School of Dental Medicine, Arietta Rigopoulos, James D. Baleja, and Addy Alt-Holland

A-3004 Effects of Oxytocin Analogs on Cellular Signaling and Neurite Outgrowth in Wild Type and OXTR-EGFP Murine Primary Hippocampal Culture

Bisini Panicker, Midwestern University, Supriya Bhuvanagiri, and Marsha L. Pierce

\section{CELL BIOLOGY}

A-3008 Investigation of Long dsRNA Mediated RNA Interference (RNAi) in Rainbow Trout

Sarah K. W. Au, Wilfrid Laurier University, and Stephanie J. DeWitte-Orr

A-3009 Tumorigenic Potential of Triclosan, an Antimicrobial Ingredient Found in Common Household Products

Anna A. Heath, Michigan State University, Jamie E. Liebold, Anthony B. Ketner, Lizbeth Lockwood, Michelle C. Schulz, and Brad L. Upham

\section{CELLULAR AND MOLECULAR BIOLOGY}

A-3010 EphA2 Gene Levels Are Increased in an In Vitro Model of Acquired Tamoxifen Resistance in Breast Cancer Olivia Godnik, Midwestern University, Hena Contractor, Kelly Keeler, and Kolla Kristjansdottir

A-3011 Investigating the Antitumor Effects of a dsRNA-Nanoparticle Complex in an In Vitro Ovarian Cancer Model Aaron Lewis, Wilfrid Laurier University, and Stephanie J. DeWitte-Orr

\section{METABOLIC ENGINEERING}

A-3012 Regulation of Fat Absorption by Peptoanaerobacter stomatis In Vivo and In Vitro

Luis Fred Badang, Midwestern University, Peymaun Mozaffari, Julia Moore, and Kristina Martinez-Guryn

\section{ONCOLOGY}

A-3013 Arsenic Trioxide Treatment Reduces Protein Levels of Mutant A NPM1 and Induces Apoptosis in NLF Neuroblastoma Cells

Oveyaa Vignesh, Midwestern University, Kelly Keeler, and Kolla Kristjansdottir

\section{ANIMAL SILENT ABSTRACT}

A-3014 Heat Shock Protein 70 \& Obesity Resistance: Interplay with Lipid Metabolism

Tori Shock, Midwestern University, Julia Moore, Matthew Pytynia, Mae Ciancio, and Kristina Martinez-Guryn

\section{ANIMAL SYMPOSIA ABSTRACTS}

\section{ORGANOIDS: UNDERSTANDING AND DEVELOPING} HUMAN DISEASE MODELS - Monday, June 7, 2021, 3:30 pm - 5:00 pm

\section{A-11}

Microphysiological Models of the Gut to Investigate Epithelial Physiology. S. MAGNESS. University of North Carolina at Chapel Hill \& North Carolina State University. Joint Departements of Biomedical Engineering. 111 Mason Farm Rd. CB\#7032, Chapel Hill, NC, 27599. Email: magness@med.unc.edu

Advances in culture of primary intestinal and colonic epithelium as organoids in many ways has tranformed in vitro study of gut biology. While organoid technology is a powerful resource, the requirement of organoids to be cultured in 3-dimensional hydrogels, the limited access to the enclosed apical tissue surface, and random tissue patterning in organoids presents a number of challenges that negatively impact efficient culture evaluation, quantificaiton and throughput. Here we present a summary of new technological advances based on microphysiological device developement for clonal organoid analysis, microinjection of cargos into the organoid lumen, and conversion of organoids to functional 2D monolayer tissues that can be used to study barrier function, nutirent handling, and mpact of microbial toxins. We then demonstrate an efficeint new platform for transgenesis and CRISP-gene editing on 2D monlayer of intestinal and colonic stem cells and marry these methods wtih a microfabricated 'planr crypt' device to show the ablity to zonate intestinal tissues into stem cell zones and differentiated cell zones. While examples of these new technologies and methods here use primary human stem cells, the platforms are highly applicable to many stem celldriven cutlure systems using other tissues. 
MODULATING THE GUT MICROBIOTA: APPLICATION OF PREBIOTICS AND PROBIOTICS FOR HUMAN HEALTH - Tuesday, June 8, 2021, $11: 15 \mathrm{pm}-1: 15 \mathrm{pm}$

\section{A-14}

The Pleiotropic Effects of Prebiotic Galacto-Oligosaccharides on the Aging Gut. M. ANDREA AZCARATE-PERIL. University of North Carolina, Dept of Medicine GI Division, School of Medicine, 332 Isaac Taylor Hall - Campus Box 7555, Chapel Hill, NC 27599-7555. Email: andrea_azcarateperil@med.unc.edu

Prebiotic galacto-oligosaccharides (GOS) have an extensively demonstrated beneficial impact on intestinal health. In this study, we determined the impact of GOS diets on hallmarks of gut aging: microbiome dysbiosis, inflammation, and intestinal barrier defects ("leaky gut"). We also evaluated if shortterm GOS feeding influenced how the aging gut responded to antibiotic challenges in a mouse model of $C$. difficile infection. Old animals had a distinct microbiome characterized by increased ratios of non-saccharolytic versus saccharolytic bacteria and, correspondingly, a lower abundance of $\beta$ galactosidases compared to young animals. GOS reduced overall diversity, increased abundance of specific saccharolytic bacteria (species of Bacteroides and Lactobacillus), increased the abundance of $\beta$-galactosidases in young and old animals, and increased non- saccharolytic organisms; however, a robust, homogeneous bifidogenic effect was not observed. GOS reduced age-associated increased intestinal permeability and increased $M U C 2$ expression and mucus thickness in old mice. Higher serum levels of IL-17 and IL-6 were observed in control and GOS diets in the antibiotics groups, and within those groups, levels of IL-6 were higher in the GOS groups, regardless of age, and higher in the old compared to young animals in the control diet groups. RTqPCR revealed significantly increased gene expression of TNF $\alpha$ in distal colon tissue of old mice, which was decreased by the GOS diet. Colon transcriptomics analysis of mice fed GOS showed increased expression of genes involved in small molecule metabolic processes and specifically the respirasome in old animals, which could indicate an increased oxidative metabolism and energetic efficiency. In young mice, GOS induced expression of binding-related genes. The galectin gene Lgals $1, \beta$-galactosyl-binding lectin that bridges molecules by their sugar moieties and is an important modulator of the immune response, and the PI3K-Akt and ECM-receptor interaction pathways were also induced in young mice. From this study we concluded that GOS modulated homeostasis of the aging gut by promoting changes in microbiome composition and host gene expression, which was translated into decreased intestinal permeability and increased mucus production. Age was a determining factor on how prebiotics impacted the microbiome and expression of intestinal epithelial cells, especially apparent from the induction of galectin-1 in young but not old mice.

\section{ANIMAL CONTRIBUTED PAPER SESSION ABSTRACT}

\section{IN VITRO ANIMAL CELL SCIENCES CONTRIBUTED PAPER SESSION - Monday, June 7,} $5: 15 \mathrm{pm}-6: 30 \mathrm{pm}$

\section{A-1007}

Antiviral Innate Immune Responses Induced by DsRNANanoparticle Complexes Inhibit Human Coronavirus Infections In Vitro. STEPHANIE J. DEWITTE-ORR, Tamiru N. Alkie, and Shawna L. Semple. Department of Health Sciences, Wilfrid Laurier University, Waterloo, CANADA. Email: sdewitte-orr@wlu.ca

Coronaviruses are RNA viruses that can cause illness in humans; the symptoms may range from the mild common cold to more severe respiratory diseases. The 2019 novel coronavirus, SARS-CoV-2, that causes COVID-19, is currently impacting human health on a global scale. In addition to important vaccine deployment, antiviral therapeutics are another piece of the puzzle to treat coronavirus infections. A novel antiviral therapeutic approach is to harness the innate antiviral immune response in the respiratory tract. Innate immunity is broad spectrum, quickly activated and exceptionally effective at inhibiting virus replication, particularly with a prophylactic treatment. Recently we have identified a nanoparticle (NP) delivery system for immunostimulatory molecules that can induce stronger innate immune responses and effectively limit virus replication without exacerbating inflammation. In the present study the efficacy of dsRNA, which induces type I interferons in vertebrate species, delivered by these phytoglycogen NPs was tested as a potential agent to limit human coronaviruses. We tested the anti-coronavirus properties of the dsRNA-NPs complex in an interferoncompetent human embryonic lung cell line and in differentiated primary human bronchial tracheal epithelial cells cultured in inserts to mimic respiratory conditions in vivo. The results of this study indicate that depending on the cell type, the combination of dsRNA and nanoparticle could either completely block virus replication or suppress coronavirus replication significantly in an interferon dependent manner. These results suggest that dsRNAnanoparticle complexes are a viable candidate for a novel, broad spectrum antiviral therapeutic, effective at limiting human coronavirus replication. 


\section{ANIMAL POSTER ABSTRACTS}

\section{A-3000}

Inhibition of TMA Production by (epi)catechin and Their Gutderived Metabolites. LISARD IGLESIAS-CARRES, Lyric K. Ramsue, Gabriella P. Agnello, Kathryn C. Racine, and Andrew P. Neilson. Plants for Human Health Institute, Department of Food, Bioprocessing and Nutrition Sciences, North Carolina State University, Kannapolis, NC. Email: liglesi@ncsu.edu

Choline is metabolized by gut bacteria to trimethylamine (TMA), which is further metabolized by the host into the pro-atherogenic metabolite trimethylamine $\mathrm{N}$-oxide (TMAO). There is interest in using dietary bioactives to reduce TMAO formation and lower atherosclerosis risk. Our objective was to study the potential of (epi)catechin and their microbial-derived metabolites (MDMs) as TMA production inhibitors in an ex vivo-in vitro fermentation model. Choline- $\mathrm{d}_{9}(100 \mu \mathrm{M})$ was fermented over $12 \mathrm{~h}$ under anaerobic conditions at $37^{\circ} \mathrm{C}$ and in $2 \%$ human fecal slurry (OpenBiome). Catechin, epicatechin and their MDMs were individually tested at $2 \mathrm{mM}(n=4)$. Potential cytotoxicity was evaluated by monitoring optical density $(600 \mathrm{~nm})$. TMA-d ${ }_{9}$ and choline- $\mathrm{d}_{9}$ levels were analyzed by UPLC-ESI-MS/MS every $2 \mathrm{~h}$. Compared to controls, the majority of treatments reduced choline- $\mathrm{d}_{9}$ metabolism at some time points, particularly at $8 \mathrm{~h}$. While inhibition of TMA-d 9 production by tested compounds was not as potent, most compounds reduced TMA-d9 production, especially at $8 \mathrm{~h}$. For most compounds, those effects were not explained by a cytotoxic effect, suggesting other inhibitory mechanisms. Generally, MDMs bioactivities were lower than the native compounds. The non-cytotoxic compounds that maintained inhibition of choline- $\mathrm{d}_{9}$ use and production of TMA- $\mathrm{d}_{9}$ throughout the full fermentation $(12 \mathrm{~h}$ ) were catechin, epicatechin and the MDM 3,4-dihydroxybenzoic acid. Our results demonstrate that catechin and epicatechin inhibit choline- $\mathrm{d}_{9}$ utilization and its microbial transformation into TMA- $\mathrm{d}_{9}$. These compounds warrant evaluation as TMA and TMAO production inhibitors in vivo.

\section{A-3001}

Impacts of Plyinosinic/Polycytidylic Acid and Novel Phytoglycogen-based Nanoparticles on Immune Defense Agents and Receptors in Human Models. KRISTOF JENIK $^{1}$, Tamiru Alkie ${ }^{1}$, Megan Dutcher ${ }^{1}$, Emily Moore ${ }^{2}$, Jondavid Dejong ${ }^{2}$, and Stephanie J. DeWitte-Orr ${ }^{1}{ }^{1}$ Dept. of Biology, Wilfrid Laurier University, Waterloo, ON, CANADA and ${ }^{2}$ Glysantis Inc, Guelph, ON, CANADA. Email: kristofjenik@gmail.com
Recently, there has been interest in the use of nanoparticles as antiviral agents as well as delivery agents for immune stimulants. Polyinosinic: polycytidylic acid (poly I:C) is a doublestranded RNA immunostimulant. Since most viruses produce dsRNA during their replicative cycle, the host cell mounts a potent immune response upon exposure. The current project focused on using a novel phytoglycogen nanoparticle (NP) as a carrier for poly I:C, to test whether it enhanced poly I:C's immuno-stimulatory effects and protected cells from infection. The impacts of poly I:C $+\mathrm{NP}$ and free poly I:C on the cell were characterized in vitro in human embryonic lung (HEL 299) cells. The protective effects of poly I:C + NP and free poly I:C against virus infection were measured using an antiviral assay. HEL 299 cells were pretreated with poly I:C + $\mathrm{NP}$ as well as free poly I:C then infected with vesicular stomatitis virus expressing green fluorescent protein (VSVgfp). Significantly more protection was measured in poly I:C+NP treated cells compared with poly I:C alone. HEL 299 cells stimulated with poly I:C + NP also expressed higher levels of interferon-beta (IFN-beta), C-X-C motif chemokine ligand 10 (CXCL10) and interferon-stimulated gene 15 (ISG15) compared to free poly I:C alone at $6 \mathrm{~h}, 24 \mathrm{~h}, 48 \mathrm{~h}$. The effects of poly I:C + NP at the protein level were also determined by immunocytochemistry. Thus, the use of poly I:C + NP should be further examined as it serves as a promising antiviral agent.

\section{A-3002}

2D Colonoid Transwell Model of Human Colonic Adenoma. KATERYNA KARPOFF ${ }^{1 *}$, CAROLINE L. MCCARTHY ${ }^{1 *}$, Ashley J. Cuttitta ${ }^{1}$, Margaret S. Bohm ${ }^{1}$, Michael K. Dame ${ }^{1}$, and Jason R. Spence ${ }^{1,2}$. ${ }^{1}$ Department of Internal Medicine, The Division of Gastroenterology, The University of Michigan Medical School, Ann Arbor, MI and ${ }^{2}$ Department of Cell and Developmental Biology, The University of Michigan Medical School, Ann Arbor, MI. *These authors contributed equally to this work. Email: kkarpoff@umich.edu, clmcc@umich.edu

The epithelial barrier of the human colon has apical and basolateral membrane domains which form a tight protective barrier that prevents luminal contents such as microbiota from crossing the epithelium. When the epithelium is perturbed, it can form a colonic adenoma, which is a precancerous lesion. In order to study the colonic epithelium and colonic barrier function, we and others use primary patient derived tissue grown in 3Dimensional (3D) organoid cultures and seeded on a 2Dimensional (2D) transwell monolayer. Here we aimed to recapitulate a precancerous condition of the colon by creating $2 \mathrm{D}$ transwell monolayers from adenoma tissue (66-year-old female with variant mutations in APC, ATM, EP300 and MSH2). To measure barrier function and assess monolayer confluence, transepithelial electrical resistance (TEER) was recorded, and tight junctions were visualized with immunofluorescence. Gene 
expression of proliferation markers was assessed using qPCR and compared to that of normal transwell monolayers as well as to adenoma and normal 3D colonoids. TEER values were lower in adenoma monolayers relative to values typical of normal monolayers, however adenoma monolayers sustained barrier TEER values for a longer duration. A proliferation marker, MKI67, was observed in the adenoma monolayer up to nine days after treatment with media that favors epithelial differentiation, in contrast to its disappearance in normal monolayers by days two or three. The cell adhesion marker, E-cadherin, was found to be disorganized and present in the cytoplasm of adenoma cells despite being primarily localized to the basolateral membrane of normal monolayers. In sum, our data demonstrates that monolayer transwell models of human adenoma manifest clinical phenotypes, including increased proliferation, dysregulated epithelial organization and reduced TEER, suggesting this is an outstanding model for direct functional studies associated with the onset of colorectal cancer.

\section{A-3003}

An In Vitro System for Maturation and Ovulation of African Catfish Ovarian Follicles. NEVENA KITANOVIĆ, Zoran Marinović, Quyen Ngoc Nguyen, Tamás Müller, Balázs Kovács, Béla Urbányi, Gergely Bernáth, and Ákos Horváth. Hungarian University of Agriculture and Life Sciences, Department of Aquaculture, Páter Károly u. 1, 2100 Gödöllö, HUNGARY. Email: nevena.n.kitanovic@gmail.com

In vitro maturation (IVM) of oocytes is a reproductive technology that enables mature eggs to be produced ex vivo, without the need for prior hormonal treatment of females. In fish, gonadotropins and maturation-inducing hormones, such as $17 \alpha, 20 \beta$-dihydroxy-4-pregnen-3-one (DHP), were shown to induce maturation of isolated ovarian follicles, although such follicles often did not ovulate in culture. Here we report successful in vitro maturation and ovulation of follicles isolated from African catfish, Clarias gariepinus (Burchell 1822). Fully grown, postvitelogenic follicles were incubated in $90 \%$ Leibovitz L-15 medium supplemented with 5\% FBS, 100 $\mathrm{U} / \mathrm{ml}$ penicillin and $100 \mu \mathrm{g} / \mathrm{ml}$ streptomycin $(\mathrm{pH} 7.6,285$ $\mathrm{mmol} / \mathrm{kg}$ ), which maintained their viability and responsiveness to hormones during treatments. Resumption of meiosis and maturation was evaluated by scoring the percentage of follicles that underwent germinal vesicle breakdown (GVBD) and ooplasm clearing. By adding DHP to the culture medium, we were able to induce GVBD in a time-dependent manner, with the highest percentage of GVBD (88\%) observed after $12 \mathrm{~h}$. Prolonged incubation did not lead to ovulation. Pre-treatment and co-incubation with human chorionic gonadotropin, insulin-like growth factor-1 and activin A had no discernible effect on GVBD. To stimulate ovulation, prostaglandins $\mathrm{F}_{2 \alpha}\left(\mathrm{PGF}_{2 \alpha}\right)$ and $\mathrm{E}_{2}\left(\mathrm{PGE}_{2}\right)$ were added to the incubation media containing DHP at different time points, and the integrity of the follicular layer was monitored throughout. Both prostaglandins were able to induce ovulation in a concentration- and time-dependent manner, with a higher percentage of ovulated oocytes present in the $\mathrm{PGF}_{2 \alpha}$ group (54\%), compared to $\mathrm{PGE}_{2}(15 \%)$. In addition, ovulation was greater in groups where the $\mathrm{PGF}_{2 \alpha}$ was added after oocytes have fully matured, which implies the stage-sensitivity of this process. In vitro techniques developed in this study can serve in aiding artificial reproduction of this, or closely related catfish species, as well as a useful system for studying oogenesis and folliculogenesis in fish.

\section{A-3004}

Effects of Oxytocin Analogs on Cellular Signaling and Neurite Outgrowth in Wild Type and OXTR-EGFP Murine Primary Hippocampal Culture. BISINI PANICKER ${ }^{1}$, Supriya Bhuvanagiri ${ }^{1}$, and Marsha L. Pierce ${ }^{2}$. ${ }^{1}$ Chicago College of Osteopathic Medicine, Midwestern University, Downers Grove, IL, 60515 and ${ }^{2}$ Department of Pharmacology, College of Graduate Studies, Midwestern University, Downers Grove, IL 60515.Email: bpanicker65@midwestern.edu, mpierc1@midwestern.edu

Oxytocin (OT) and Vasopressin (VP) have various physiological, social, and behavioral functions in the peripheral and central nervous system. Perturbation in OT and OT receptor expression are associated with disorders such as autism, schizophrenia, and depression. OT has a short half-life and poor penetration of the blood brain barrier, and clinical trial results have been variable. In this study, we used the consensus mammalian OT, endogenous to both human and mouse, with a leucine in the $8^{\text {th }}$ position (Leu ${ }^{8}-\mathrm{OT}$ ) and the two ana$\log \mathrm{s}$ with a proline in the $8^{\text {th }}$ position $\left(\mathrm{Pro}^{8}\right.$-OT and $\mathrm{Val}^{3}-\mathrm{Pro}^{8}$ OT), which have longer half-lives. Both OT receptor and vasopressin 1a (V1a) receptor are expressed in murine hippocampi, so primary hippocampal cultures were generated from embryonic day 18 murine hippocampi. Transgenic OXTREGFP mice that express GFP under control of the OT receptor promoter were used to examine the effects on neuronal morphology. OXTR-EGFP cultures did not display GFP expression, potentially due to prenatal culturing. Functional assays were used to assess OTR and V1aR activation in wild-type primary hippocampal cultures by observing the fluorescence when Fluo-8 (an indicator dye) was bound to intracellular calcium. At 12 days in vitro, Leu ${ }^{8}$-OT and Pro ${ }^{8}$-OT analogs displayed an increase in oscillation amplitude with increasing OT analog dose, which may be due to signaling through the V1a receptor. Further analysis with OT receptor and V1a receptor inhibitors will confirm receptor signaling pathways. Organotypic hippocampal cultures might provide a better model for examining neurite outgrowth. Overall, evaluating 
the effects of these analogs should help us gain a better understanding of their molecular mechanisms and guide us in developing new and effective therapeutic agents.

\section{A-3005}

Evaluation of the Effects of Cadmium on NRK-52E, HK-2, and RPTEC Proximal Tubule Renal Epithelial Cells. MILONI PATEL ${ }^{1}$, Kelly Keeler ${ }^{1}$, Taulant Besiri ${ }^{1}$, Rachel Arceneaux ${ }^{1}$, Heshaam Latifi ${ }^{2}$, Vishal Patel ${ }^{1}$, Joshua R. Edwards $^{3}$, Walter C. Prozialeck ${ }^{3}$, and Michael J. Fay ${ }^{3}$. ${ }^{1}$ Department of Biomedical Sciences, Midwestern University, Downers Grove, IL; ${ }^{2}$ Chicago College of Osteopathic Medicine, Midwestern University, Downers Grove, IL; and ${ }^{3}$ Department of Pharmacology, Midwestern University, Downers Grove IL. Email: mfayxx@midwestern.edu,mpatel15@midwestern.edu

Cadmium is an important environmental pollutant that is toxic to humans. Once in the body, cadmium complexes are filtered at the glomerulus and reabsorbed by the proximal tubule renal epithelial cells. When a critical threshold concentration occurs in these cells, nephrotoxicity develops. Although cadmium-induced nephrotoxicity has been widely studied, the molecular alterations that lead to kidney injury are not fully elucidated. Cultured proximal tubule epithelial cells offer an in vitro research model to study cadmium-induced toxicity at the cellular and molecular levels. The purpose of this study is to compare three proximal tubule epithelial cell lines in their responses to cadmium. These cell lines are rat NRK-52E, human HK2, and human RPTEC. To determine the effect of cadmium on cell viability, the cell lines were exposed to cadmium $(0$, $1,2.5,5,10,15,20,25,50,75,100 \mu \mathrm{M})$ for $24 \mathrm{~h}$. After $\mathrm{Cd}$ exposure, cells were incubated with alamarBlue ${ }^{\mathrm{TM}} \mathrm{HS}$ cell viability reagent for $3 \mathrm{~h}$. Cadmium induced a dosedependent decrease in the viable cell number with $\mathrm{IC}_{50}$ values of $18.3 \mu \mathrm{M}, 31.9 \mu \mathrm{M}$ and $9.7 \mu \mathrm{M}$ for NRK-52E, Hk-2 and RPTEC, respectively. We are also looking at cadmium-induced expression of the biomarker Kidney Injury Molecule-1 (KIM-1) mRNA. Studies in the NRK52E cell line indicate that $\mathrm{Cd}$ caused a 9.8-, 13.9- and 9.8fold significant increase at $15 \mu \mathrm{M}, 20 \mu \mathrm{M}$ and $25 \mu \mathrm{M}$, respectively. Cadmium-induced expression of KIM-1 mRNA in the HK-2 and RPTEC cell lines is currently under investigation. These studies indicate that NRK-52E, HK-2, and RPTEC cell lines are responsive to the toxic effects of cadmium and may serve as valuable in vitro research models for studying the cellular/molecular alterations associated with cadmium nephrotoxicity. We are currently using these cell lines to study the role of microRNAs in cadmium-induced kidney injury.

\section{A-3006}

Exploring DsRNAi in Human Cells as a Potential Antiviral Therapy for Coronaviruses. SHAWNA L. SEMPLE and Stephanie J. DeWitte-Orr. Department of Health Sciences and Biology, Wilfrid Laurier University, Waterloo, ON, CANADA. Email: ssemple@wlu.ca

To oppose the persistent exposure to viruses, humans have evolved effective innate antiviral immune responses that are based on the type I interferon (IFN) system. Long doublestranded RNA (dsRNA), is a potent inducer of IFNs and is produced by almost all viruses during replication. The intensity of the dsRNA-induced IFN response is dependent on dsRNA length, but not sequence. Another dsRNA-mediated antiviral response is the RNAi pathway. While intact and functioning in vertebrate cells, the role of RNAi as an antiviral response using long dsRNA (dsRNAi) remains contentious. This study provides evidence that dsRNAi is not only functional, but has a strong antiviral effect in IFN-competent human fibroblasts (THF), human lung cells (MRC5) and human cancer cells (glioblastoma, SNB75) towards the rhabdovirus vesicular stomatitis virus expressing green fluorescent protein (VSV-GFP) and the human coronavirus, HCoV-229E. When both THF and SNB75 were pre-soaked with GFP sequencespecific long dsRNA at concentrations previously shown to be too low to induce IFN, a significant reduction in the $\mathrm{TCID}_{50}$ of VSV-GFP was observed when compared to the mismatched sequence controls. This reduction in viral titre was associated with length of the sequence-specific dsRNA, as knockdown was not observed when shorter dsRNA molecules were used. Additionally, soaking with GFP siRNA was unable to reduce VSV-GFP numbers until transfection methods were used. When both cell lines were pre-soaked with dsRNA containing VSV viral gene sequences ( $\mathrm{N}$ and $\mathrm{M}$ protein), a significant reduction in viral titre was also observed. To confirm that this was not only a phenomenon of VSV-GFP, HCoV-229E was also examined. MRC5 were pre-soaked with viral gene dsRNA followed by viral infection. Though RdRp dsRNA did not provide protection, $\mathrm{N}, \mathrm{M}$ and $\mathrm{S}$ protein dsRNA significantly reduced $\mathrm{HCoV}-229 \mathrm{E}$ titres. The results provide the first evidence that soaking with gene-specific dsRNA is capable of generating viral knockdown in vertebrate cells. Long dsRNAi is currently an untapped platform for developing human antiviral therapeutics.

\section{A-3007}

The Effect of Epithelial-Stromal Cross-talk on Tumor Cell Metabolism in Basal Cell Carcinoma Development. TATIANA MENDEZ ${ }^{1}$, Arietta Rigopoulos ${ }^{1}$, James D. Baleja $^{2}$, and Addy Alt-Holland ${ }^{1} .{ }^{1}$ Tufts University School of 
Dental Medicine, One Kneeland Street, Boston, MA 02111 and ${ }^{2}$ Tufts University School of Medicine, 136 Harrison Avenue, Boston, MA 02111. Email: tatiana.mendez@ tufts.edu

The development of numerous skin basal cell carcinomas (BCC) is one of the main clinical manifestations of Gorlin syndrome patients. While aberrant activation of the sonic hedgehog pathway drives BCC development, the cross-talk between epidermal BCC cells and dermal fibroblasts, the tumor microenvironment, and reprogramming of tumor energy metabolism can also contribute to the growth of these tumors. We have shown that BCC cells and fibroblasts have distinct metabolic signatures when grown in individual cultures or in mixed BCC/fibroblast co-cultures. In the latter, both direct BCC-fibroblast interactions, and indirect cross-talk between these two cell types, could affect the culture metabolic activity. To define the effect of the indirect cell-cell cross-talk on the metabolism of these cells, we established advanced BCC/ fibroblast co-cultures in which $\mathrm{BCC}$ cells and fibroblasts were placed in separate compartments in an insert/tray system. This allowed for indirect cell-cell cross-talk only between the two cell types in the same culture. Cell morphology was analyzed by microscopy, and metabolomics was used to analyze the levels of lactate, pyruvate, glutamate, and glutamine in individual $\mathrm{BCC}$ and fibroblast cultures and advanced $\mathrm{BCC} /$ fibroblast co-cultures. In all culture settings, BCC cells exhibited a variety of shapes and sizes, while fibroblasts exhibited uniform spindle morphology. When grown in individual cultures, fibroblasts secreted more lactate and glutamate, and consumed more pyruvate and glutamine than BCC cells. However, advanced BCC/fibroblast co-cultures showed reduced secretion of lactate and glutamate, and reduced consumption of pyruvate and glutamine. This indicates a strong effect that BCC cells can exert on the metabolism of fibroblasts through indirect BCC-fibroblast cross-talk. In turn, the changes in the overall metabolic activity of the cells can create a favorable microenvironment to support tumor cell growth, and further contribute to continuous development of $\mathrm{BCC}$ tumors in Gorlin syndrome patients.

\section{A-3008}

Investigation of Long dsRNA Mediated RNA Interference (RNAi) in Rainbow Trout. SARAH K. W. AU and Stephanie J. DeWitte-Orr. Department of Biology, Wilfrid Laurier University, Waterloo, ON, CANADA. Email: auxx5290@mylaurier.ca, sdewitteorr@wlu.ca

RNA interference (RNAi) was first characterized in plants and invertebrates as an antiviral innate immune response. It is a natural antiviral defence mechanism to degrade viral RNA by virusinduced gene silencing. Studies showed synthetic long double- stranded RNA (dsRNA; >30bp) degraded Caenorhabditis elegans messenger RNA (mRNA) transcripts, resulting in sequence-dependent gene silencing. Currently, there is only limited research on RNAi in fish, particularly with the use of long dsRNA to trigger sequence-specific gene silencing and prevent viral infection. This is because long dsRNAs trigger interferon responses and upregulate interferon stimulated gene (ISG) expression that masks gene-specific RNAi effects in vertebrates. This study explores long dsRNA-mediated RNAi pathways in rainbow trout cells from two aspects. Firstly, the optimal dsRNA concentration that would funnel into RNAi without triggering type 1 interferon responses was determined. Secondly, the potential of sequence-specific dsRNA to mediate gene silencing and protect against viral infection was identified. Luciferase sequence specific, long dsRNA (dsRNA-Luc) was able to silence induced RTG-P1 luciferase gene expression in the rainbow trout gonadal cell line, RTG-P1, while a mis-match dsRNA sequence did not. Additionally, a dsRNA-CSV sequence was able to protect the rainbow trout gonadal cell line, RTG-2, against chum salmon reovirus (CSV) infection. Long thought non-functional in vertebrates, long RNAi-based strategies could be used to study rainbow trout gene function, develop new drugs and vaccines to control infectious diseases in the future.

\section{A-3009}

Tumorigenic Potential of Triclosan, an Antimicrobial Ingredient Found in Common Household Products. ANNA A. HEATH, Jamie E. Liebold, Anthony B. Ketner, Lizbeth Lockwood, Michelle C. Schulz, and Brad L. Upham. Department of Pediatrics and Human Development, Michigan State University, East Lansing, MI 48824. Email: heathann@msu.edu

Triclosan is an antimicrobial agent that was a common ingredient in many household products such as antibacterial soaps, body washes, toothpastes and some cosmetic products. Recently triclosan has been implicated in adverse health effects such as immune suppression and liver cancer, and has been since banned by the Food and Drug Administration in 2016. However, it remains in a multitude of household items and is a tremendous public health concern. Considering that triclosan has been linked to liver cancer, there is a need to understand the underlying mechanisms of action. Cancer cells have long been characterized as cells that lose their ability to regulate growth through contact inhibition and lack the ability to terminally differentiate, which implies a breakdown in one of the communicating mechanisms. In particular, the dysregulation of gap junctional intercellular communication (GJC) is one hallmark characteristic of cancer. Thus, we determined the effects of triclosan on GJIC in a bipotent epithelial stem cell line derived from F344 rats. GJIC was measured using the scalpel load-dye transfer assay. Triclosan inhibited GJC in a dose and time dependent manner 
implicating that triclosan can contribute to the cancer process by dysregulating GJC. Preliminary data suggests triclosan does not work through a mitogen-activated protein kinase pathway, and may work partially through a phosphatidylcholine-specific phospholipase $\mathrm{C}$ pathway. Future experiments will determine if natural products, such as resveratrol and quebecol, can mitigate the adverse effects of triclosan on GJC.

\section{A-3010}

EphA2 Gene Levels Are Increased in an In Vitro Model of Acquired Tamoxifen Resistance in Breast Cancer. OLIVIA GODNIK, Hena Contractor, Kelly Keeler, and Kolla Kristjansdottir. Department of Biomedical Sciences, Midwestern University, Downers Grove IL 60515. Email: ogodnik77@midwestern.edu

Breast cancer is the most common cancer in American women. For estrogen receptor positive breast cancer patients, tamoxifen is FDA approved for both treatment and prevention. However, long-term use of tamoxifen often results in acquired resistance to tamoxifen, and if this could be avoided, studies have shown that patients may benefit from longer usage. To generate tamoxifen resistant cells in vitro, we grew MCF-7 breast cancer cells in low levels of tamoxifen for over 3 months. AR-MCF71 and AR-MCF7-2 cell lines were generated and one control cell line, MCF7-1, was generated by growing in media without tamoxifen. Next, using RT-qPCR, we examined expression levels of selected genes, estrogen receptor alpha (ESR1) and EphA2, in both the control and tamoxifen treated cells. ESR1 is commonly downregulated in tamoxifen resistant cells. Indeed, the results show a decreased trend of ESR1 expression in the cells grown long-term in tamoxifen compared to the control MCF7-1 cells. EphA2 is a receptor tyrosine kinase found to be overexpressed in breast cancer. EphA2 has been shown to transform breast epithelial cells in vitro to display properties commonly associated with the development of metastasis. Our results show that the cells grown long-term in tamoxifen, AR-MCF7-1 and AR-MCF7-2, had an increase in EphA2 expression compared to the control MCF7-1 cells. Our results suggest that long term exposure to tamoxifen can lead to gene expression changes consistent with tamoxifen resistance and aggressive breast cancer.

\section{A-3011}

Investigating the Antitumor Effects of a dsRNA-Nanoparticle Complex in an In Vitro Ovarian Cancer Model. AARON LEWIS $^{1}$ and Stephanie J. DeWitte-Orr ${ }^{1,2}$. ${ }^{1}$ Department of Biology, Wilfrid Laurier University, Waterloo, CANADA and ${ }^{2}$ Department of Health Science, Wilfrid Laurier University, Waterloo, CANADA. Email: lewi6160@mylaurier.ca
An estimated 1 in 75 women will be diagnosed with ovarian cancer in their lifetime. Despite advanced detection and treatment methods, it remains a silent killer with an expected survival rate of $50 \%$. A developing method in cancer treatment is the use of compounds that stimulate the immune system to aid in the body's fight against the disease. This project focused on the use of double-stranded RNA (dsRNA), commercially available polyinosinic:polycytidylic acid, poly(I:C) specifically, as a potent immune stimulant to induce cytotoxicity in two ovarian cancer cell lines; SKOV-3 and OVCAR-3. Some challenges exist with the delivery of dsRNA due to its large size and negative charge. One way to overcome this hurdle is the use of nanoparticles to deliver the dsRNA to the cell. Thus, a phytoglycogen nanoparticle $(\mathrm{PhG})$ was used as a carrier for poly(I:C). In this study, poly(I:C) conjugated to $\mathrm{PhG}$ (polyIC$\mathrm{PhG}$ ) was found to induce significant cell death in both SKOV-3 and OVCAR-3 but not in healthy ovarian cell controls IOSE-386 72h post-treatment. PolyIC-PhG induced cell death was induced at concentrations up to four times less than poly IC alone. Cisplatin is a chemotherapy drug used to treat ovarian cancer. PolyIC-PhG also enhanced cisplatin-induced cell death in SKOV-3 and OVCAR-3, with greater effect than poly IC alone. Next, the immunogenicity of dsRNA-induced cell death was investigated. At earlier time points, PolyIC$\mathrm{PhG}$ induced cell death produced calreticulin presentation on the cell surface and expression of CXCL10, an innate immune protein, in both ovarian cancer cell lines. This data suggests that poly IC-PhG induces immunogenic cell death, ICD. Collectively this data suggests dsRNA-PhG has potential as an adjuvant therapy against ovarian cancer.

\section{A-3012}

Regulation of Fat Absorption by Peptoanaerobacter stomatis In Vivo and In Vitro. LUIS FRED BADANG, Peymaun Mozaffari, Julia Moore, and Kristina Martinez-Guryn. Department of Biomedical Science, Midwestern University Downers Grove, IL. Email: Lbadang44@midwestern.edu

Previous work has shown that microbes derived from high fat (HF) diet conditions increase lipid absorption in germ free (GF) mice, thereby establishing a direct link between diet-microbe interactions and host processes that lead to obesity. The aim of this project was to determine if mice inoculated with Peptoanaerobacter stomatis would increase host lipid absorption leading to obesity in mice fed low fat (LF) or HF diet. Our secondary goal was to examine if this specific bacterial strain induces genes involved in fat absorption in duodenal and jejunal intestinal organoids. It was hypothesized that conventional mice inoculated with $P$. stomatis will have an increase in lipid absorption regardless of the diet consumed. To achieve these goals, 11 week old mice were randomly assigned to one of four groups: a 
HF or LF diet control group or a LF or HF group supplemented with $P$. stomatis with an $\mathrm{n}=5$ per group. Mice in the LF or $\mathrm{HF}+$ $P$. stomatis groups were inoculated weekly with $1 \times 10^{\wedge} 8$ CFUs $P$. stomatis and body weight, adiposity, plasma lipids and stool lipid content were measured. In addition, duodenal organoids were treated with $10 \%$ conditioned media (CM) collected from $P$. stomatis and genes involved in lipid absorption and esterification were measured. Mice fed $\mathrm{HF}$ diet $+P$. stomatis did not exhibit a change in body weight but displayed increased retroperitoneal fat pad mass and total fat mass with concurrent decrease in fecal triglyceride (TG) levels compared to HF diet alone. Interestingly, mice on LF diet $+P$. stomatis exhibited reduced plasma TG levels compared to control. Therefore, the impact of $P$. stomatis appears to be dependent on the host diet. In the organoids it was observed that $P$. stomatis $\mathrm{CM}$ upregulated the gene synthesis of diacylglycerol acyltransferase (DGAT)2, important for TG esterification in the intestine, representing a possible mechanism through which $P$. stomatis regulates lipid absorption.

\section{A-3013}

Arsenic Trioxide Treatment Reduces Protein Levels of Mutant A NPM1 and Induces Apoptosis in NLF Neuroblastoma Cells. OVEYAA VIGNESH, Kelly Keeler, and Kolla Kristjansdottir. Department of Biomedical Sciences, College of Graduate Studies, Midwestern University, Downers Grove, IL. Email: ovignesh43@midwestern.edu, kkrist@midwestern.edu

Neuroblastoma is a pediatric solid tumor of the sympathetic nervous system. We previously found that high-risk neuroblastoma cells have elevated nucleophosmin-1 (NPM1) levels compared to low-risk cells. NPM1 is a multifunctional protein that is localized primarily to the nucleolus. A mutation of NPM1 common in acute myeloid leukemia patients, mutant A, redirects the protein to the cytoplasm. AML cells harboring mutant A NPM1 are sensitive to arsenic trioxide (ATO) which induces apoptosis and targeted degradation of the mutant A NPM1 protein. In contrast, wild-type NPM1 is not degraded following treatment with ATO in AML. To examine whether the mutant A NPM1 is reduced in levels in neuroblastoma cells, we transfected NLF neuroblastoma cells with mutant A NPM1 or wildtype NPM1. Following transfection, the cells were treated with ATO and NPM1 protein levels were analyzed via western blot. NLF cells treated with $1 \mu \mathrm{M}-3 \mu \mathrm{M}$ ATO for 48 hours had decreased levels of mutant A NPM1 protein levels compared to vehicle treated cells. However, wild-type NPM1 protein levels did not decrease following exposure to ATO. Apoptosis levels were also investigated through the cleavage of PARP via western blot. ATO treatment of NLF cells with wild-type and mutant NPM1 resulted in PARP cleavage but not in the vehicle treated cells. We are currently examining if the reduced levels of mutant A NPM1 protein are due to ubiquitin-targeted proteasome degradation and examining the mechanism of apoptosis induction.

\section{A-3014}

Heat Shock Protein 70 \& Obesity Resistance: Interplay with Lipid Metabolism. TORI SHOCK, Julia Moore, Matthew Pytynia, Mae Ciancio, and Kristina Martinez-Guryn. Biomedical Sciences Program, Midwestern University, Downers Grove IL. Email: tori.shock@midwestern.edu

With the worldwide obesity epidemic progressively worsening, identification of therapeutic targets against this condition has become increasingly important. Recent unpublished studies have demonstrated dietinduced obesity resistance in mice with intestinespecific overexpression of Heat Shock Protein 70 (HSP70), which is conserved across eukaryotes and important for reduction of cellular stress. However, the mechanism behind this phenotype remains unclear. The goal of this research was to determine if altered lipid absorption is the cause of the observed obesity resistance. Thus, mice containing a villin-specific HSP70 transgene (HSP70Tg mice) were fed a low fat (LF) or high fat (HF) diet for 12 weeks and mRNA levels of genes involved in fat absorption in the jejunum were measured. Separate sets of mice were used to collect fasting lipids and conduct radiolabeled lipid absorption assays using H3-labeled triolein (triglyceride composed of oleic acid). First, it was found that HSP70Tg mice displayed significantly lower LDL and total cholesterol levels in fasting plasma compared to non-transgenic (Ntg) mice fed HF diet. In addition, HSP70Tg mice on HF diet exhibited significantly decreased gene expression of Dgat2, Cd36, and SCD1 compared to Ntg mice in the jejunum. HSP70Tg and Ntg mice were gavaged with $\mathrm{H}^{3}$-labeled triolein delivered in $200 \mathrm{ul}$ olive oil 10 minutes after receiving $10 \%$ tyloxapol injection to prevent peripheral uptake of the ingested lipid. Plasma samples were collected at 1, 3, 5, and 7 hours post gavage and following euthanasia, small intestinal mucosal scrapings from the mice were harvested. While there were no detectable differences in plasma or stool H3-triolein levels, HSP70Tg mice exhibited significantly reduced levels in duodenal and jejunal mucosal scrapings compared to Ntg mice. Taken together, the current findings of this research suggest that obesity resistance in HSP70Tg mice may be due to altered lipid absorption and metabolism pathways in the small intestine, but further investigation is needed to establish the exact mechanisms involved. 


\section{INDEX}

\begin{tabular}{|c|c|c|c|c|c|}
\hline Agnello, Gabriella P. & A-3000 & Fay, Michael J. & A-3005 & Moore, Emily & A-3001 \\
\hline Alkie, Tamiru & A-3001 & Godnik, Olivia & A-3010 & Moore, Julia & A-3012 \\
\hline Alkie, Tamiru N. & A-1007 & Heath, A. & A-3009 & Moore, Julia & A-3014 \\
\hline Alt-Holland, Addy & A-3007 & Horváth, Ákos & A-3003 & Mozaffari, Peymaun & A-3012 \\
\hline Arcenaux, Rachel & A-3005 & Igless-Carres, Lisard & A-3000 & Müller, Tamás & A-3003 \\
\hline Au, Sarah K. W. & A-3008 & Jenik, Kristof & A-3001 & Nguyen, Quyen Ngoc & A-3003 \\
\hline Azcarate-Peril M. Andrea & A-14 & Karpoff, Kateryna & A-3002 & Nielson, Andrew P. & A-3000 \\
\hline Badang, Luis Fred & A-3012 & Keeler, Kelly & A-3005 & Panicker, Bisini & A-3004 \\
\hline Baleja, James D. & A-3007 & Keeler, Kelly & A-3010 & Patel, Miloni & A-3005 \\
\hline Bernáth, Gergely & A-3003 & Keeler, Kelly & A-3013 & Patel, Vishal & A-3005 \\
\hline Besiri, Taulant & A-3005 & Ketner, Anthony B. & A-3009 & Pierce, Marsha L. & A-3004 \\
\hline Bhuvanagiri, Supriya & A-3004 & Kovács, Balázs & A-3003 & Prozialeck, Walter C. & A-3005 \\
\hline Bohm, Margaret S. & A-3002 & Kitanović, Nevena & A-3003 & Pytnia, Matthew & A-3014 \\
\hline Ciancio, Mae & A-3014 & Kristjansdottir, Kolla & A-3010 & Racine, Kathryn C. & A-3000 \\
\hline Contractor, Hena & A-3010 & Kristjansdottir, Kolla & A-3013 & Ramsue, Lyric K. & A-3000 \\
\hline Cuttitta, Ashley J. & A-3002 & Latifi, Heshaam & A-3005 & Rigopolulos, Arietta & A-3007 \\
\hline Dame, Michael K. & A-3002 & Lewis, Aaron & A-3011 & Schultz, Michelle C. & A-3009 \\
\hline Dejong, Jondavid & A-3001 & Liebold, Jamie E. & A-3009 & Semple, Shawna L. & A-1007 \\
\hline DeWitte-Orr, Stephanie J. & A-1007 & Lockwood, Lizbeth & A-3009 & Semple, Shawna L. & A-3006 \\
\hline DeWitte-Orr, Stephanie J. & A-3001 & Magness, S. & A-11 & Shock, Tori & A-3014 \\
\hline DeWitte-Orr, Stephanie J. & A-3006 & Marinović, Zoran & A-3003 & Spence, Jason R. & A-3002 \\
\hline DeWitte-Orr, Stephanie J. & A-3008 & Martinez-Guryn, Kristina & A-3012 & Upham, Brad L. & A-3009 \\
\hline DeWitte-Orr, Stephanie J. & A-3011 & Martinez-Guryn, Kristina & A-3014 & Urbányi, Béla & A-3003 \\
\hline Dutcher, Megan & A-3001 & McCarthy, Caroline L. & A-3002 & Vignesh, Oveyaa & A-3013 \\
\hline Edwards, Joshua R. & A-3005 & Mendez, Tatiana & A-3007 & & \\
\hline
\end{tabular}

